\title{
Comparative study of dexmedetomidine and clonidine as an adjunct to levobupivacaine in transversus abdominis plane block in patients undergoing total abdominal hysterectomy: a randomized control study
}

\author{
Bharati Gupta $^{1}$, Amit Gupta ${ }^{2}$, R. K. Verma ${ }^{1}$, Payal Shah ${ }^{1 *}$
}

\author{
${ }^{1}$ Department of Anesthesia, Dr. RPGMC Tanda at Kangra, Himachal Pradesh, India \\ ${ }^{2}$ Department of Obstetrics and Gynecology, Dr. RPGMC Tanda at Kangra, Himachal Pradesh, India
}

Received: 17 July 2019

Accepted: 07 September 2019

\author{
*Correspondence: \\ Dr. Payal Shah, \\ E-mail: payalshah0179@gmail.com
}

Copyright: () the author(s), publisher and licensee Medip Academy. This is an open-access article distributed under the terms of the Creative Commons Attribution Non-Commercial License, which permits unrestricted non-commercial use, distribution, and reproduction in any medium, provided the original work is properly cited.

\begin{abstract}
Background: Ultrasound guided TAP block is safe, simple and effective method of providing postoperative analgesia in surgeries involving abdominal wall incision by blocking anterior branches of thoracolumbar nerves originating from T6-L1. Our aim to study the efficacy of dexmedetomidine and clonidine as an adjunct to levobupivacaine in ultrasound guided TAP block for postoperative analgesia in patients undergoing TAH.

Methods: Prospective, double blind randomized control study. Ninety ASA I and II patients scheduled for TAH were randomly assigned in a double blinded study and divided into three groups. Group L received $18 \mathrm{ml}$ of $0.25 \%$ levobupivacaine $+2 \mathrm{ml}$ of NS to make total volume of $20 \mathrm{ml}$ on each side. Group LC and Group LD received $18 \mathrm{ml}$ of $0.25 \%$ levobupivacaine $+1 \mathrm{mcg} / \mathrm{kg}$ of clonidine or dexmedetomidine diluted in NS to make total volume of $20 \mathrm{ml}$ on each side. USG guided TAP block was given when subarachnoid block level regressed to T10 level. Postoperatively patients were assessed for pain scores, HR, SBP, DBP, nausea vomiting, sedation and satisfaction scores at 0, 2, 4, 6 and 12 and 24 hours. Statistical analysis was performed using SPSS software 17. p value $<0.05$ was considered significant.

Results: Pain scores were significantly lower in LD and LC groups as compared to L group and demand for first rescue analgesic was delayed in LD group $(491.50 \pm 73.29 \mathrm{~min})$ and group LC $(268.00 \pm 35.47 \mathrm{~min})$ as compared to group L $(129.17 \pm 10.67 \mathrm{~min})$. The total number of demand doses in 24 hours were significantly less in group LD $(1.00 \pm 0.00)$ followed by group LC $(2.03 \pm 0.18)$ and group L $(2.77 \pm 0.57)$ respectively. Incidence of hypotension, bradycardia and sedation was more in LD group as compared to LC and L groups.

Conclusions: TAP block with dexmetomidine as an adjunct to levobupivacaine provides prolonged postoperative analgesia as compared to clonidine as an adjunct and plain levobupivacaine.
\end{abstract}

Keywords: Clonidine, Dexmedetomidine, Hysterectomy, Levobupivacaine, Transversus abdominis plane block

\section{INTRODUCTION}

The transversus abdominis plane (TAP) Block is a type of local anaesthetic block that is being increasingly used as part of multimodal analgesia in surgeries involving the anterior and lateral abdominal wall. With recent advancements in ultrasound technology, TAP block has become a "must-know" technique for postoperative pain management for such surgeries. ${ }^{1}$ It involves the deposition of local anaesthetic in the plane between the 
internal oblique and transversus abdominis muscles targeting the spinal nerves originating from the T6 to L1 spinal roots, thus interrupting sensory nerve supply to the anterolateral abdominal wall including abdominal skin, muscles and parietal peritoneum. ${ }^{2}$ Recent evidence supports the effectiveness of TAP blocks for a variety of abdominal surgeries, such as cholecystectomy, colectomy, cesarean section, hysterectomy, prostatectomy, and hernia repair. ${ }^{3-5}$

The advantages of TAP block as compared to different regional anesthesia include preservation of lower limb motor-sensory function, hemodynamic stability, and less invasiveness. ${ }^{6}$

Post hysterectomy pain is largely parietal pain inflicted by abdominal incision and visceral pain caused by organ manipulation and trauma during surgery. ${ }^{7}$ Multimodal strategies play an important role for analgesia may mitigate opioid side effects, including postoperative nausea and vomiting (PONV) and sedation.

Opioid-sparing effects of TAP block when used as part of multimodal drug therapy in patients undergoing total abdominal, laparoscopic, or vaginal hysterectomy, performed under general or spinal anesthesia or both have been studied. Tubog et al concluded that, TAP block has moderate opioid-sparing effects up to 48 hours after hysterectomy. Likewise, patients who underwent TAP blocks had fewer opioid-related side effects. ${ }^{8}$

Unfortunately, TAP block duration is limited to the effect of administered LA.

Levobupivacaine and bupivacaine has similar potency but levobupivacaine seems advantageous in terms of its lower risk of cardiovascular and CNS toxicity, less negative ionotropic effect and causing less QT prolongation.

Recently, local anesthetic adjuvants like dexmedetomidine, clonidine and dexamethasone have been the subject of increasing interest as the potential to prolong blockade duration. ${ }^{-11}$ The combined use of a local anesthetic agent and adjuvants when, applied in a TAP block, may help to evolve an ideal protocol for pain control after abdominal surgery.

Some meta-analyses indicated that perineural dexmedetomidine can prolong the durations of sensory block and motor block as well as analgesia when administered in brachial plexus block. ${ }^{12}$ Unlike brachial plexus block, TAP block is a nondermatomal "field block," which requires a large volume of anesthetics to cover several spinal nerves.

Addition of clonidine $1 \mu \mathrm{g} / \mathrm{kg}$ to $20 \mathrm{ml}$ bupivacaine $0.25 \%$ in TAP block bilaterally for cesarean section significantly increases the duration of postoperative analgesia, decreases postoperative analgesic requirement, and increases maternal comfort compared to $20 \mathrm{ml}$ of bupivacaine $0.25 \%$ alone.

To the authors' knowledge, there are no published metaanalyses investigating the comparative effect of dexmedetomidine and clonidine as adjuvants in TAP blocks for postoperative pain. This study was designed to determine the efficacy of dexmedetomidine and clonidine as local anesthetic adjuvant in prolonging the duration of TAP blocks.

\section{METHODS}

After approval by institutional ethics committee and written informed consent, 96 ASA I-II female patients in the age group 35-70 years with BMI 18.5-29.9, scheduled for TAH through pfannenstiel incision under subarachnoid block were enrolled for the study for a period of 18 months. Exclusion criteria were patient refusal, patients with a history of cardiac, respiratory, renal or hepatic and coagulation disorders, local site infection, psychological disorders, allergy to study medications. The patients were allocated by random number chart and the study drug solution was prepared by a nonparticipant staff. Group $\mathrm{L}(\mathrm{n}=32)$ received $18 \mathrm{ml}$ of $0.25 \%$ levobupivacaine $+2 \mathrm{ml}$ of NS each side, Group LC $(n=33)$ and Group LD $(n=31)$ received $18 \mathrm{ml}$ of $0.25 \%$ levobupivacaine $+1 \mathrm{mcg} / \mathrm{kg}$ clonidine or dexmedetomidine $+\mathrm{NS}$ (total volume $=20 \mathrm{ml}$ ) each side.

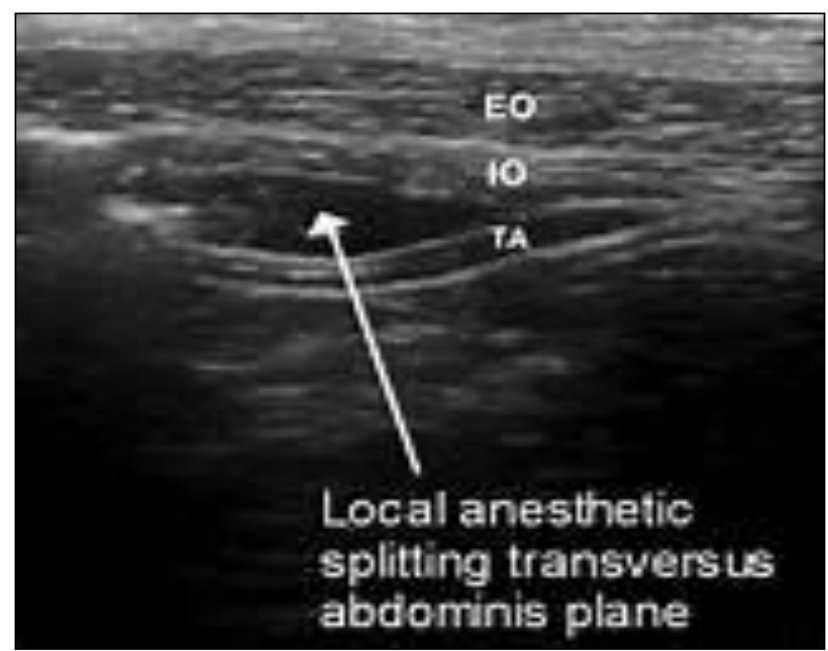

EO: External oblique, IO: Internal oblique, TA: Transverse abdominis.

Figure 1: Ultrasound-guided TAP Block with needle tip placement and drug spread.

Patients undergoing TAH were given subarachnoid block under all aseptic conditions. After confirmation of T6 level, surgery was started. After the surgery, when the subarachnoid block level regressed to T10, ultrasound guided TAP block was given. After drapping the abdominal part, using linear probe $(6-13 \mathrm{MHz}$ frequency), $23 \mathrm{G}$ spinal needle was advanced by in-plane technique at the anterior axillary line. After checking the exact 
location of the needle tip $20 \mathrm{ml}$ of drug solution was given. Intramuscular diclofenac $1.5 \mathrm{mg} / \mathrm{kg}$ was administered to all patients after the surgery was over, as per the protocol of institution. For the first 24 hours intramuscular diclofenac given on demand when pain scores exceeded 4 but not more than 3 doses in 24 hours. In patients with persistent pain intravenous tramadol 100 $\mathrm{mg}$ given as a second rescue analgesic.

The patients were asked to rate average pain over 24 hours postoperatively on visual analogue scale and to the severity of nausea, vomiting on a four point scale. HR, SBP, DBP and sedation scores were monitored at 0, 2, 4, 6, 12 and 24 hours postoperatively. The patients were asked to rate on a 3-points scale their satisfaction with pain management.

Ninety patients thirty in each group completed the study successfully.

\section{Statistical analysis}

Statistical analysis was performed using SPSS software 17. The One Sample Kolmogorov-Smirnov Test was employed to determine whether data sets differed from normal distribution. Normally distributed data was analyzed using a repeat-measures general linear model analysis of variance (ANOVA). For comparison between two groups post hoc test was applied in normally distributed data. The Bonferroni correction was used to correct for multiple testing at different time points.

"p"value was taken as level of significance:

$\mathrm{p}>0.05$ Not significant, $\mathrm{p}<0.05$ Significant, $\mathrm{p}<0.01$ highly significant, $\mathrm{p}<0.001$ Very highly significant.

\section{RESULTS}

After approval by institutional ethics committee and written informed consent, ninety six ASA I-II female patients in the age group 35-70 years were recruited in the study. Six patients were excluded because of block failure. Thereby ninety patients thirty in each group completed the study successfully. The mean age, weight, duration of surgery and ASA status were comparable in all the three groups and the difference was statistically insignificant $(\mathrm{p}>0.05)$ (Table 1$)$.

Table 1: Demographic profile.

\begin{tabular}{|llllll|}
\hline Parameter & Group L $(n=30)$ & Group LC $(\mathbf{n = 3 0})$ & Group LD $(\mathbf{n = 3 0})$ & $\mathrm{p}$-value \\
\hline Age $($ years) Mean \pm SD & $43.80 \pm 6.39$ & $45.07 \pm 6.76$ & $45.07 \pm 6.21$ & 0.682 \\
\hline Weight $(\mathrm{kg})$ Mean \pm SD & & $55.67 \pm 7.36$ & $56.87 \pm 8.21$ & $55.37 \pm 6.56$ & 0.710 \\
\hline Duration of surgery (min) Mean \pm SD & $93.17 \pm 15.61$ & $93.67 \pm 16.76$ & $92.50 \pm 15.57$ & 0.961 \\
\hline \multirow{2}{*}{ ASA grade $(\%)$} & I & $23(76 \%)$ & $18(60 \%)$ & $25(83 \%)$ & 0.132 \\
\cline { 2 - 6 } & II & $7(24 \%)$ & $12(40 \%)$ & $5(17 \%)$ & \\
\hline
\end{tabular}

$\mathrm{P}$ value $<0.05$ (significant).

Table 2: Comparison of mean duration of analgesia (time to first analgesic requirement).

\begin{tabular}{|c|c|c|c|}
\hline Group & Duration in min (Mean \pm SD) & p-value & Sig diff between two groups \\
\hline Group L & $129.17 \pm 10.67$ & \multirow{3}{*}{0.000} & $\mathrm{~L}$ and $\mathrm{LC}-\mathrm{P}=0.000$ \\
\hline Group LC & $268.00 \pm 35.47$ & & $\mathrm{~L}$ and $\mathrm{LD}-\mathrm{P}=0.000$ \\
\hline Group LD & $491.50 \pm 73.29$ & & $\mathrm{LC}$ and $\mathrm{LD}-\mathrm{P}=0.000$ \\
\hline
\end{tabular}

Lower mean VAS scores were seen in group LD at 2 hours and 12 hrs, in LC group at 6 hours and in group L at 4 hours and 24 hours (Figure 2).

Lower HR was seen after 2 hours with LD $(64.63 \pm 3.02)$ group and LC $(67.93 \pm 2.99)$ group as compared to $\mathrm{L}$ group (71.53 \pm 2.71$)$.

Lower SBP was seen after 2 hours of TAP block with LD $(111.60 \pm 5.73)$ and LC (114.73 \pm 5.69$)$ groups as compared to group L (117.27 \pm 5.44$)$.

Lower DBP was seen after 2 hours of TAP block with LD $(71.41 \pm 4.45)$ and LC $(74.37 \pm 3.78)$ groups as compared to group L $(76.73 \pm 4.21)$.

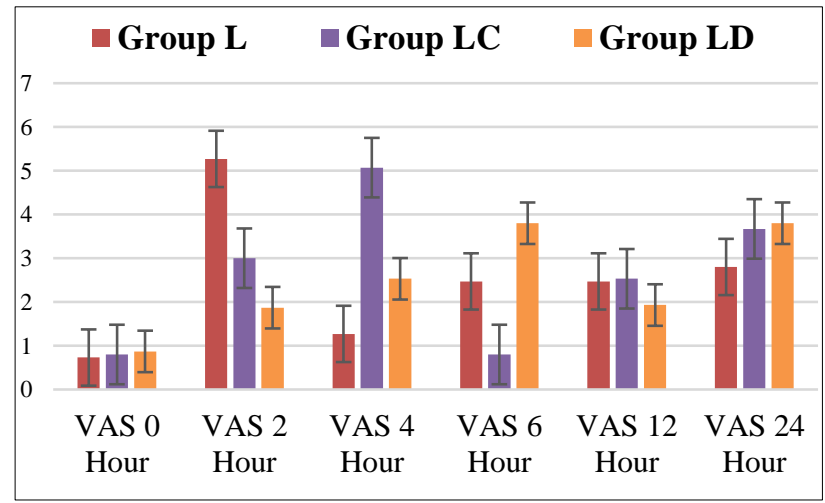

Figure 2: Comparison of VAS score in patients undergoing TAH. 
Mean duration of analgesia that is duration to first analgesic requirement was found to be significantly prolonged in both group LD (491.50 \pm 73.29$)$ and group LC (268.00 \pm 35.47$)$ compared to group B $(129.17 \pm 10.67)$ $(\mathrm{P}<0.05)$ (Table 2).
The total number of demand doses in 24 hours were significantly less in group LD $(1.00 \pm 0.00)$ followed by group LC $(2.03 \pm 0.18)$ and group L $(2.77 \pm 0.57)$ respectively with $\mathrm{p}<0.05$ (Table 3 ).

Table 3: Comparison of total number of rescue demands in 24 hours.

\begin{tabular}{|ll|ll|}
\hline Group & Total number of demands in 24 hours (Mean \pm SD) & p-value & Sig diff between two groups \\
\hline Group L & $2.77 \pm 0.57$ & \multirow{2}{*}{0.000} & L and LC- $\mathrm{P}=0.000$ \\
\hline Group LC & $2.03 \pm 0.18$ & L and LD - P $=0.000$ \\
\hline Group LD & $1.00 \pm 0.00$ & LC and LD $-\mathrm{P}=0.000$ \\
\hline
\end{tabular}

Higher mean PONV score was recorded in group L $(1.10 \pm 0.31)$ followed by group LC $(1.03 \pm 1.00)$ and group LD $(1.03 \pm 0.18)$ respectively (Figure 3 ).

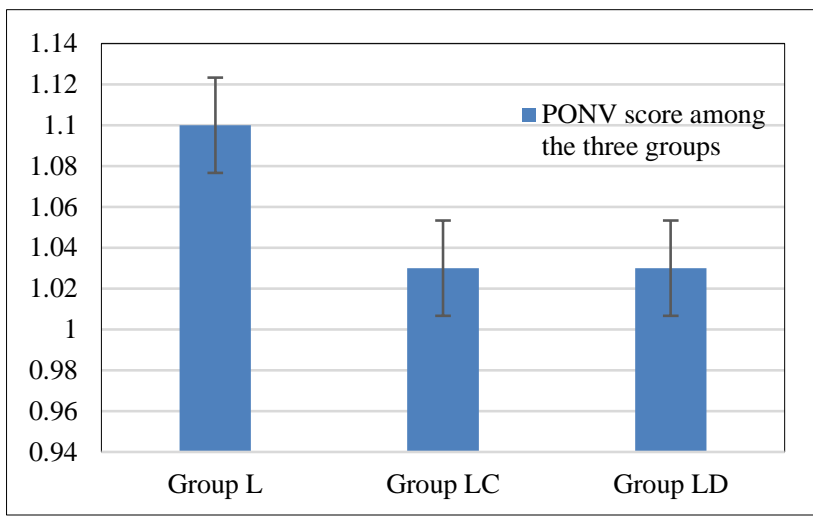

Figure 3: PONV score among the three groups.

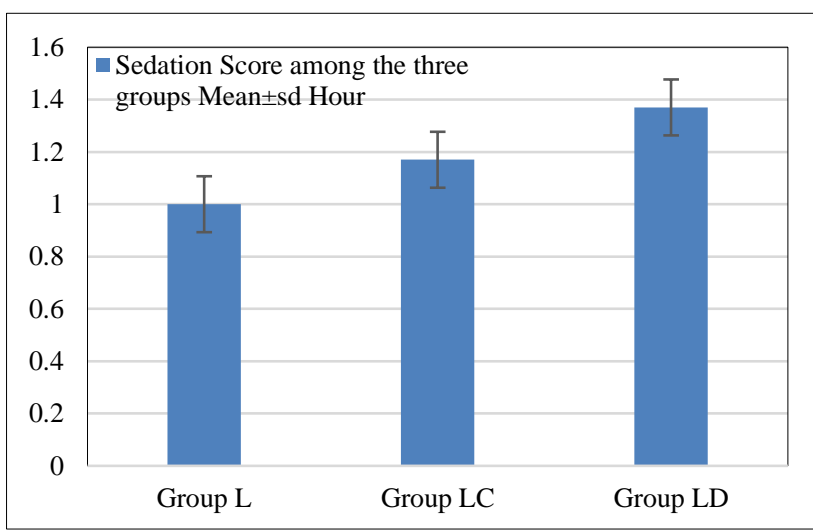

Figure 4: Sedation score among the three groups.

Sedation score was recorded in group LD (1.30 \pm 0.47$)$, group LC (1.00 \pm 0.00$)$ and group L $(1.00 \pm 0.00)$ and was found to be higher in group LD as compare to group LC and group L (Figure 4).

Satisfaction score was recorded in group LD (1.03 \pm 0.18$)$, group LC (1.03 \pm 0.18$)$ and group L $(1.57 \pm 0.63)$ and was found to be better in group $\mathrm{LD}$ and group $\mathrm{LC}$ as compared to group L (Figure 5).

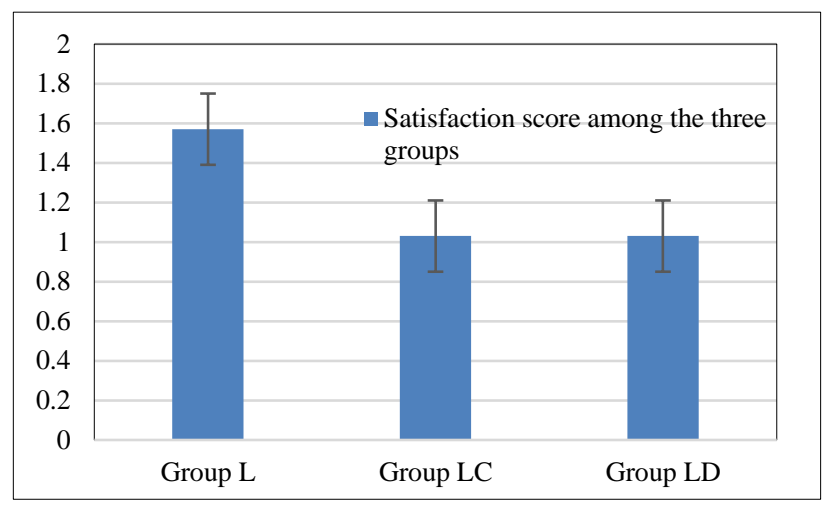

Figure 5: Satisfaction score among the three groups.

\section{DISCUSSION}

Transversus abdominis plane (TAP) block is a relatively new regional anesthetic technique used to provide postoperative analgesia of the anterolateral abdominal wall for various abdominal surgeries. It is an effective component of multimodal analgesic regimen for a wide variety of abdominal procedures. Over time, the type and concentration of local anaesthetic used for TAP block has been changed. Doses were increased to provide prolonged postoperative analgesia but at the expense of increasing the dose of local anesthetics and so side effects. So another way to prolong the analgesic effect is to add adjuncts to local anesthetic solution, in concentrations recommended for other peripheral blocks. Various adjuvants have been studied in TAP block like $\alpha 2$ agonists, dexamethasone, magnesium and epinephrine. But out of these the analgesic effect of $\alpha 2$ agonists like dexmedetomidine and clonidine is well known. Therefore, we intended to use $\alpha 2$ agonists as a adjuvants to levobupivacaine.

Statistically lower HR was seen between 0 to 2 hours with dexmedetomidine group as compared to clonidine group. Similar to our study Almarakbi and Kaki also found lower HR at $60 \mathrm{~min}$ which continued till 4 hours postoeratively with dexmedetomidine as compared to plain bupivacaine. ${ }^{13}$ 
Statistically lower SBP was seen between 0 to 2 hours with dexmedetomidine group as compared to clonidine group. Similar to our study Swami SS and colleagues in 2012 in supraclvicular brachial plexus block also found significant reduction in SBP and DBP in dexmedetomidine group as compared to clonidine group.

The mean duration of analgesia with clonidine $(1 \mathrm{mcg} / \mathrm{kg})$ was $268 \mathrm{~min}$ and with dexmedetomidine $(1 \mathrm{mcg} / \mathrm{kg})$ was 491 min. Similar to our study Almarakbi and Kaki have also found same mean duration of analgesia with dexmedetomidine group. Total number of demands for rescue analgesia, were significantly least with dexmetomidine followed by clonidine and maximum in plain levobupivacaine group. Similar to our study Abdelaal and collegues also observed lesser number of demamd doses with dexmedetomidine group as compared to plain levobupivacaine group. ${ }^{14}$ In our study the nausea vomiting score was comparable in all the three groups. Sedation score was found to be higher in dexmedetomidine group as compared to clonidine group and plain levobupivacaine group. Similar to our study Almarakbi and Kaki in 2014 also found higher sedation score with dexmedetomidine group as compared to bupivacaine group.

Statistically significant difference was found in terms of satisfaction score and better satisfaction score was found in groups with clonidine and dexmedetomidine as compared to levobupivacaine group.

\section{CONCLUSION}

To summarize, addition of dexmedetomidine and clonidine as an adjunct to levobupivacaine in TAP block provides prolonged postoperative analgesia as compared to plain group. Among dexmedetomidine and clonidine dexmetomidine has more prolonged analgesia as compared to clonidine group.

\section{Funding: No funding sources}

Conflict of interest: None declared

Ethical approval: The study was approved by the Institutional Ethics Committee

\section{REFERENCES}

1. Abdallah F, Chan WV, Brull R. Transversus abdominis plane block: A systemic review. Reg Anesth Pain Med. 2012;37(2):193-209.

2. Rozen WM, Tran TMN, Ashton MW, Barrington MJ, Ivanusic JJ, Taylor GI. Refining the course of the thoracolumbar nerves: A new understanding of the innervation of the anterior abdominal wall. Clin Ana. 2008;21(4):325-33.
3. Champaneria R, Shah L, Geoghegan J, Gupta JK, Daniels JP. Analgesic effectiveness of transversus abdominis plane blocks after hysterectomy: A metaanalysis. Eu J Obstet Gynecol Repro Biol. 2013;166(1):1-9.

4. Elkassabany N, Ahmed M, Malkowicz SB, Heitjan DF, Isserman JA, Ochroch EA. Comparison between the analgesic efficacy of transversus abdominis plane (TAP) block and placebo in open retropubic radical prostatectomy: A prospective, randomized, doublelinded study. J Clin Anesth. 2013;25(6):459-65.

5. Peng K, Ji FH, Liu HY, Wu SR. Ultrasound-guided transversus abdominis plane block for analgesia in laparoscopic cholecystectomy: a systematic review and meta-analysis. Med Prin Pract. 2016;25(3):237-46.

6. Petersen PL, Mathiesen O, Torup H, Dahl JB. The transversus abdominis plane block: A valuable option for postoperative analgesia? A topical review. Acta Anaesthesiol Scand. 2010;54(5):529-35.

7. Cervero F, Laird JM. Visceral pain. Lancet. 1999;353(9170):2145-8.

8. Tubog TD. Opioid-sparing effects of transversus abdominis plane block in elective hysterctomy: a systematic review and meta-analysis. AANA J. 2018;86(1):41-55.

9. Brummett CM, Hong EK, Janda AM. Perineural dexmedetomidine added to ropivacaine for sciatic nerve block in rats prolongs the duration of analgesia by blocking the hyperpolarization-activated cation current. Anesthesiol. 2011;115(4):836-43.

10. El-Boghdadly K, Brull R, Sehmbi H. Perineural dexmedetomidine is more effective than clonidine when added to local anesthetic for supraclavicular brachial plexus block: a systematic review and meta-analysis. Anesth Analg. 2017;124:2008-20.

11. Hussain N, Grzywacz VP, Ferreri CA. Investigating the efficacy of dexmedetomidine as an adjuvant to local anesthesia in brachial plexus block: a systematic review and meta-analysis of 18 randomized controlled trials. Reg Anesth Pain Med. 2017;42:184-96.

12. Singh R, Kumar N, Jain A, Joy S. Addition of clonidine to bupivacaine in transversus abdominis plane block prolongs postoperative analgesia after cesarean section. J Anaesthesiol Clin Pharmacol. 2016;32(4):501-4.

13. Almarakbi WA, Kaki AM. Dexmedetomidine plus bupivacaine in TAP block. Saudi J Anaesth. 2014;8(2):161-6.

14. Abdelaal W, Metry AA, Refaat M, Ragaei M, Nakhla G. Comparative study between levobupivacaine versus levobupivacaine plus dexmedetomidine for transverse abdominis plane block in postoperative pain management after abdominoplasty. Enliven: J Anesthesiol Crit Care Med. 2015;2(2):004.

Cite this article as: Gupta B, Gupta A, Verma RK, Shah P. Comparative study of dexmedetomidine and clonidine as an adjunct to levobupivacaine in transversus abdominis plane block in patients undergoing total abdominal hysterectomy: a randomized control study. Int J Reprod Contracept Obstet Gynecol 2019;8:3991-5. 